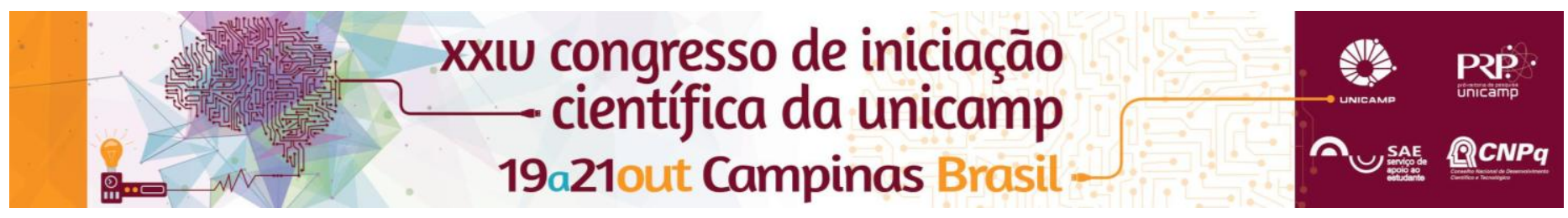

\title{
Achados multiparamétricos de ressonância magnética em massas anexiais
}

\author{
Ana Carolina B. Silva*, Patrick N. Pereira, Sophie F. M. Derchain, Luis O. Sarian, Adriana Yoshida, Karla Araújo
}

\begin{abstract}
Resumo
Objetivo: avaliar a eficácia do sistema ADNEX MR SCORING System para diagnóstico pré-operatório de massas anexiais. Sujeitos e Métodos: O comite de Ética da instituição aprovou esse estudo prospectivo. Os exames de Ressonância Magnética (RM) foram realizados em 108 pacientes com 136 massas anexiais identificadas no período entre Março de 2014 a Dezembro de 2015. Os exames de RM foram realizados por dois radiologistas com 6 e 7 anos de experiência, respectivamente, em RM da pelve. As imagens da RM foram classificadas de acordo com ADNEX MR SCORING desenvolvido por Thomassin-Naggara et al (2013). Chi-quadrado, testes de comparação múltiplos de Tukey e coeficiente Kappa foram utilizados para análise estatística. Resultados: Das 68 massas benignas, o mais frequente foi neoplasia epitelial, seguido dos tumores de células germinativas. Endrometrioma foi a lesão benigna não neoplásica mais frequente. Entre os 68 tumores malignos, cistadenocarcinoma de ovário foi o mais prevalente. Aplicando o algorítimo de Thomassin-Naggarae et al. (2008) a sensibilidade para malignidade dos escores 4 ou 5 foi de $95.4 \%$ e a especificidade foi de $94.1 \%$.O critério morfológico mais associado à malignidade foi lesão sólida, com valor preditivo positivo de 12.3. Conclusão: O ADNEX MR SCORING demonstrou alta acurácia compatível com os resultados revelados por Thomassin-Naggara. A concordância quase perfeita entre os radiologistas demonstraram que o estudo é altamente reproduzível.
\end{abstract}

Palavras-chave: Massas anexiais, câncer de ovário, Ressonância Magnética, diagnóstico pré-operatório.

\section{Introdução}

A Ultrassonografia transvaginal (USGTV) é utilizado para avaliação de massas anexiais suspeita de neoplasia. 20\% das massas são indeterminadas ao USGTV e dessas, $80 \%$ são benignas. Pela RM $92 \%$ das massas anexiais categorizados como indeterminados a USGTV são adequadamente classificados (1). Novos parâmetros funcionais na avaliação por RM das lesões ovarianas estão sendo desenvolvidos e aperfeiçoados, destacando-se a análise temporal (estudo dinâmico) do padrão de realce da lesão pelo contraste paramagnético EV e mais recentemente a utilização de sequências ponderadas em difusão. Thomassin-Naggaraet al.2008(2). Há escassa produção científica que correlaciona diretamente os achados da RM o diagnóstico das massas anexiais. Assim, o objetivo desse estudo é avaliar a eficácia do sistema ADNEX MR SCORING System para diagnóstico pré-operatório de massas anexiais.

\section{Resultados e Discussão}

Das 68 massas benignas, o mais frequente foi neoplasia epitelial, seguido dos tumores de células germinativas. Endrometrioma foi a lesão benigna não neoplásica mais frequente. Entre os 68 tumores malignos, cistadenocarcinoma de ovário foi o mais prevalente. Foram encontrados 13 tumores ovarianos borderlines. Aplicando o algorítimo de Thomassin-Naggarae et al. (2008) a sensibilidade scores 4 ou 5 foi de $95.4 \%$ e a especificidade de $94.1 \%$. Os três falsos negativos foram tumores borderlines descritas como cistos multiloculares sem componente sólido e realce da parede por contraste endovenoso. O critério morfológico mais associado à malignidade foi lesão sólida, com valor preditivo positivo de 12.3. Houve concordância quase perfeita (índice Kappa $=0.91$ ) para a classificação final das lesões pelo ADNEX MR scoring system entre os radiologistas
Figura 1. Fluxograma correlacionando resultados patológicos, score final no ADNEX MR e achados patológicos.

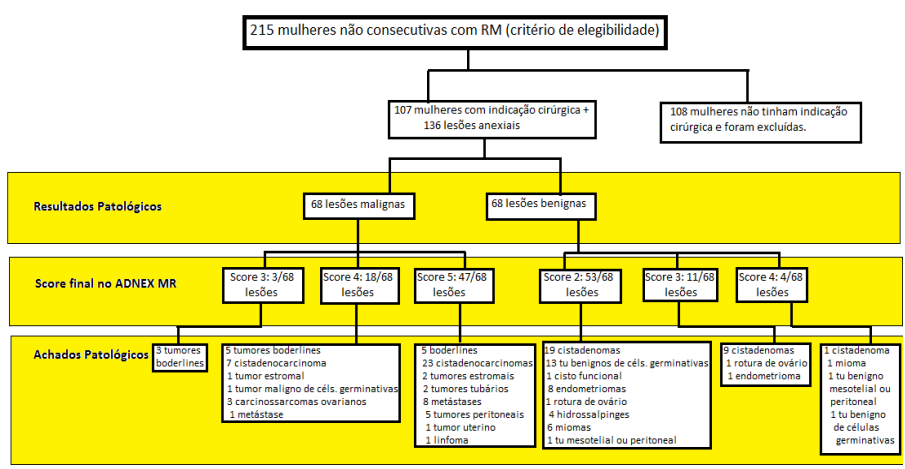

\section{Conclusões}

O ADNEX MR SCORING demonstrou alta acurácia compatível com os resultados revelados por ThomassinNaggara. A concordância quase perfeita entre os radiologistas demostraram que o estudo é altamente reproduzível.

\section{Agradecimentos}

Centro de Atenção Integrada à Saúde da Mulher CAISM. Fundação de Amparo à pesquisa do Estado de São Paulo - FAPESP

1. Sohaib SA, Mills TD, Sahdev A, Webb JAW, VanTrappen PO, Jacobs IJ, et al. The role of magnetic resonance imaging and ultrasound in patients with adnexal masses. Clin Radiol. março de 2005;60(3):340-8.

2. Thomassin-Naggara I, Bazot M, Daraï E, Callard P, Thomassin J, Cuenod CA. Epithelial ovarian tumors: value of dynamic contrast-enhanced MR imaging and correlation with tumor angiogenesis. Radiology. julho de 2008;248(1):148-59. 